\title{
Human Organisms from an Evolutionary Perspective: Its Significance for Medicine
}

\author{
Mahesh Ananth
}

\section{Contents}

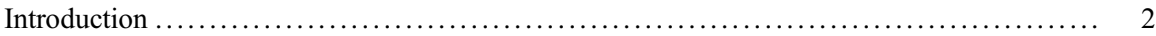

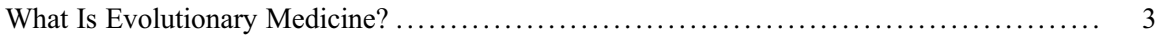

The Human Body as an Evolutionary Entity: The Compromised Physical Self ............ 3

Relevance to Medical Teaching and Practice .................................. 4

Biological Function ..................................................... 5

Reply to Evolutionary Functionalism ....................................... 8

Replies to Etiological Evolutionary Functionalism .............................. 9

Replies to Propensity Evolutionary Functionalism ............................. 11

Benefits to Medicine ..................................................... 13

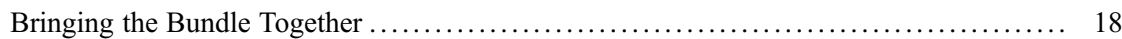

The Evolved Bundled-Homeostatic Body: Influence on the Practice of Medicine and Public

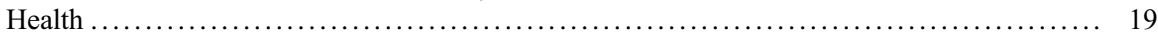

Defense Mechanism: The Fever ........................................ 19

Genetic Disease and PHJs: Sickle-Cell Anemia .............................. 21

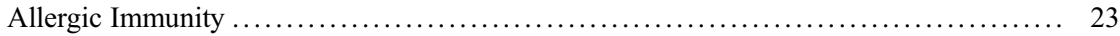

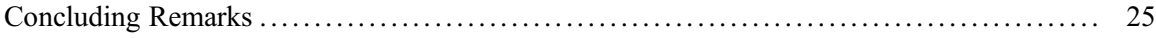

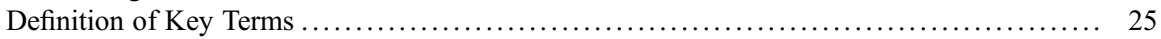

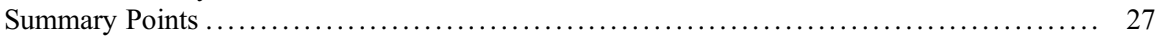

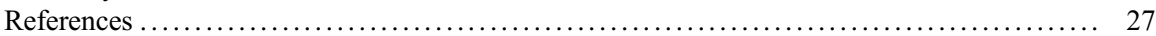

\begin{abstract}
Defenders of evolutionary medicine claim that medical professionals and public health officials would do well to consider the role of evolutionary biology with respect to the teaching, research, and judgments pertaining to medical theory and practice. An integral part of their argument is that the human body should be understood as a bundle of evolutionary compromises. Such an appreciation,
\end{abstract}

\footnotetext{
M. Ananth $(\bowtie)$

Department of Philosophy, Indiana University South Bend, South Bend, IN, USA

e-mail: mananth@iusb.edu
} 
which includes a proper understanding of biological function and physiological homeostasis, would provide a crucial perspective regarding the understanding and securing of human health needs currently lacking in the medical arena.

\section{Introduction}

Geneticist and evolutionary biologist Theodosius Dobzhansky (1973, p. 125) famously decreed, "Nothing in biology makes sense except in the light of evolution." This near-oracular pronouncement has echoed throughout the discipline of biology, even extending to other non-biology-related subject matters (Mayr 2000). Still, the full effect of evolutionary thinking with respect to medicine is waiting to be felt. One straightforward reason for this lag is a time horizon difference. On the one hand, medical practitioners are concerned with caring for the human body based on an individual/personal history time frame (ontogeny), and public health officials focus their efforts around producing the social infrastructure that both mitigates the spread of disease and secures health benefits that pertain to individuals, populations, and communities in the short run (see APHA 2016, url, and Hartig et al. 2014). On the other hand, evolutionary biologists examine species/bodies/traits under the guise that their current existence owe to advantages gained via a more or less geologic timescale perspective (phylogeny) (Ewald 1994, p. 8). Still, granting that humans are the product of a long history of evolutionary processes, it is reasonable to explore the significance of humans as one among the animals for medicine (i.e., evolutionary/ Darwinian medicine) in a way that can supplement the perspective of medical practitioners and public health officials. Indeed, there is no hubris here on the part of the defenders of evolutionary medicine. Rather, proponents of evolutionary medicine acknowledge that the activities and achievements of medicine reveal a history of stellar insights, which have drawn upon solid scientific practices and reflection. They also counsel that one more accoutrement to the already impressive "medical toolkit" could very well engender unanticipated boons (World Health Organization, 2007, url).

This chapter offers an analysis of some of the key philosophical and biological issues pertaining to the role that evolutionary biology can play within the theoretical and practical aspects of medicine. To this end, section II will address the nature and scope of evolutionary medicine. Section III will explicate the idea of the body as a bundle of evolutionary compromises and the relevance of such a notion to medicine with special attention given to four prominent concepts of biological function and two components of homeostasis. Section IV will draw upon the biological function and homeostatic aspects of the human body and propose how such a perspective can help make sense of the mechanism of fever, sickle-cell anemia, and allergic responses. 


\section{What Is Evolutionary Medicine?}

Evolutionary medicine [hereafter EM] is concerned with both biological and philosophical issues pertaining to the field of medicine and public health (WHO, url). Although it may seem unambiguously obvious, on the biological side, medicine is inexorably linked to biology. In general, this is primarily made manifest in the physical health judgments [hereafter PHJs] tendered by healthcare professionals. Inescapably, then, concepts that are germane to biology will be correspondingly relevant to PHJs. On the assumption that both medicine and public health are concerned with understanding, establishing, and improving human health, and simultaneously mitigating and controlling the effects of disease states, EM can contribute insights that may be valuable to the practices of medicine and public health on the health, disease, and public policy fronts; this includes evolutionary explanations and medical prescriptions pertaining to "defenses, infection, novel environments, genes, design compromises, and evolutionary legacies" (Nesse and Williams 1994, 1997). It is this evolutionary component that is not patently discernible, argue defenders of EM, in the biologically based PHJs made by medical practitioners and reveals why the link between biology and medicine requires further investigation. On the philosophical side, the issues range over establishing a proper understanding of the human body. This is accomplished by giving special attention to the biological concepts of function, homeostasis, and natural selection. Ultimately, the aim of EM is to provide both conceptual tools and practical insights for medical and public health professionals in their quest for improving the human condition as it relates to health and disease. Although this EM perspective is beginning to gain traction in the scholarly literature (Sprouffke et al. 2012; Alexander et al. 2014), EM advocates have been strongly urging that medical practitioners and public health officials would do well to consider more diligently evolutionary reflections as they pertain to both PHJs and the nature and modification of health and disease states in human populations.

\section{The Human Body as an Evolutionary Entity: The Compromised Physical Self}

Since maintaining and improving physical human health are integral parts of the primary goals of medicine and public health, it is crucial to have an accurate understanding of the human body from an evolutionary perspective and how this relates to the concept of health. What, then, is the human body from a Darwinian perspective? The answer is that the human body is (1) a bundle of adaptive compromises, (2) which are evolved functions that (3) have an overarching goal of concomitantly securing both internal and organism-to-environment homeostasis (4) for the sake of survival and reproductive success (Ananth 2008 and see Goldstein 
1963, for physical holism discussion). What this means is that many of the features of the human body are the product of a long geologic history of give-and-take as our species adapted to a range of environmental perturbations. From this vantage point, not only were certain features "selected for" survival and reproductive success with respect to local environments, but these very features qua whole organism were modifying the local environments creating a constant dynamic playing field (OdlingSmee et al. 2003). Thus, not only were traits functioning to help secure survival and reproductive success in local environments, but they were also doing so while maintaining a kind of dual internal compromise equilibrium and an organismenvironment-compromise equilibrium in the presence of varying degrees of environmental dynamism.

What are the implications of the human body as an evolved internal-externalequilibrium "bundle of compromises"? Nesse and Williams (1994, p. 19) succinctly provide the following answer:

Like any engineer, evolution must constantly compromise...If something works well enough that its deficiencies do not constitute a selective force, there is no way natural selection can improve it. Thus, while every part of the body has some reserve capacity to deal with occasionally encountered extreme circumstances, every part is also vulnerable when its reserve capacity is exceeded. There is nothing in the body that never goes wrong.

What can be gleaned from Nesse and Williams is that an evolutionary perspective on the body includes acknowledging that less than optimal features are an integral part of understanding the human body as a bundle of compromises. The reason for this is that adaptations are adaptations to local environments. If a feature functions suboptimally, but still performs well enough in the light of how well other features are functioning in a local environment, then its suboptimal functioning does not necessarily represent a selective disadvantage. Rather, it may very well be the case that no group occupies the fitness landscape wherein optimal fitness is possible (Gilchrist and Kingsolver 2001).

\section{Relevance to Medical Teaching and Practice}

The importance of anatomy to medicine cannot be overemphasized (Older 2004 and Papa and Vaccarezza 2013). Over the past 20 years, however, the teaching and understanding of anatomy have come under fire. On the academic side, the somewhat slow transition from lecture and cadaver dissection training/learning to technology-based training/learning has given this discipline a somewhat dull image, resulting in subpar dissemination of knowledge at both undergraduate and medical school venues. On the economic side, labs and instructors are costly resulting in a lack of infrastructure upkeep (Turney 2007 and Bergman et al. 2014). Despite the many in-house battles regarding pedagogy and funding, there appears to be some consensus that a balance must be achieved between traditional learning styles and technology-driven approaches in the face of scarce 
resources. What isn't mentioned in much of this review literature is the relevance of evolutionary thinking with respect to the teaching of anatomy and medicine in general. As it currently stands, anatomy texts tend to focus on the systems approach; that is, students are taught about the detailed workings of various systems in the body (e.g., nervous system, digestive system, endocrine system, etc.) and their mechanical part interactions with very little pedagogy regarding how these systems are really bundles of evolved interacting compromises. This supposedly stark oversight in medicine has not been lost on defenders of EM:

Evolutionary biology is so firmly integrated with the rest of biology that it is not possible to mark a boundary between them. But modern medicine has been a peninsula...From secondary school through medical school, the fundamental relevance of evolution to all of human life often has been ignored or even suppressed. (Ewald 1994, p. 7)

Both the suppression of and indifference to the role of evolutionary thinking in medicine may very well be the product of religious and political worries, but its almost-glaring omission from the field needs serious reconsideration in the eyes of most proponents of EM. To illustrate, as a way of mitigating the putative "banausic" effect of anatomy on medical students, Turney (2007) has insisted that the natural wonder associated with the workings of the human body should be given its due. Yet, much like others analyzing this recent decline in the interest in anatomy, Turney does not suggest that a "Darwinian twist" might be one of the missing elements needed to ignite a much looked-for blend of enthusiasm and creative curiosity. Defenders of EM believe this can be achieved by showing the practical implications that EM can have on PHJs and a fortiori the practices of everyday medicine. As Shanahan (1999, lecture) has suggested, "At present Darwinian medicine exists mainly at the level of theory, but the insights of Darwinian medicine could work a profound transformation in practice of medicine in the next century." Nesse and Williams (1994, p. xi) also offer their respectful recommendation to medical mavens: "We are not urging an alternative to modern medical practice but rather an additional perspective from a well-established body of scientific knowledge that has been largely neglected by the medical profession." If this transformation and/or added perspective suggested by advocates of EM happens sooner rather than later, then understanding the human body as a bundle of evolutionary compromises is a grand place to start from the EM perspective. Correspondingly, the concepts of biological function and homeostasis, which are discussed in the next two sections, also require explication in order to paint an accurate picture of the human body qua evolving bundle of compromises.

\section{Biological Function}

\section{Systemic Functionalism}

Beyond an evolutionary bundle of compromises, the previous section introduced both "biological function" and "bodily homeostasis" as integral parts of the concept 
of the human body as an evolved entity. This section provides a discussion of the nature of biological function, while the next section addresses the nature of bodily homeostasis. In the philosophical literature, there is a robust discussion, not only on the nature of biological function (Ananth 2017; Nissan 1997 and Wouters 2003a and 2003b) but also on the variety of evolutionary accounts of function with respect to health and disease (see Ananth 2008, for citations). The result is that four accounts of biological function have emerged as front runners: (1) Systemic Functionalism, (2) Etiological Evolutionary Functionalism, (3) Propensity Evolutionary Functionalism, and (4) Mixed Evolutionary Functionalism. Although debates persist on which account most plausibly reconciles philosophical worries and biological realities (Ariew et al. 2002), the "systems" account provides a point of departure. According to this view, if a component $\mathbf{X}$ causally contributes to system $\mathbf{S}$ 's performance of $\mathbf{Z}$, then $\mathbf{X}$ 's contribution constitutes a function of $\mathbf{S}$ 's performance of $\mathbf{Z}$ (Cummins and Roth 2010 and Boorse 2002). The heart, for example, is a feature of the circulatory system. This system, which includes the lungs and blood vessels, works to keep about 51 of blood continuously moving through the body in a constant exchange of waste-filled blood for oxygen-rich blood. So, the heart has the function of pumping and exchanging good and bad blood through the body because it causally contributes to the circulatory system's overall task of moving blood throughout the body. We can formally make sense of Systemic Functionalism as follows:

Systemic Functionalism: A feature $\mathbf{X}$ has a function in system $\mathbf{S}$ if and only if activity $\mathbf{Y}$ of $\mathbf{X}$ causally contributes to $\mathbf{S}$ 's overall capacity/performance of $\mathbf{Z}$.

In terms of health and disease, Boorse argues that Systemic Functionalism [hereafter $\mathbf{S F}$ ] should be favored. His rationale is that physical health and disease are restricted to the subfield of pathology, which is concerned with the proper function of parts of organisms within specific subsystems of the body (Boorse 1987; my italics). From this perspective, the thyroid is healthy when it actualizes the function of producing thyroxin in the endocrine system because it causally contributes to the endocrine system's overall capacity/performance of metabolic regulation.

It is worth noting that, much like the systems approach taken in terms of understanding anatomy, $\mathbf{S F}$ is the version of biological function endorsed by much of the medical/clinical community (see Tyreman 2001). What this means is that emphasis is given to understanding how the parts of organisms function within particular subsystems with less attention given to the subtle evolutionary give-andtake with respect to these systems and the body as a whole. For so long as the thyroid produces the appropriate amount of thyroxin in terms of its the contribution to metabolic regulation, the medical community would endorse such production as indicative of a healthy functioning thyroid (Tyreman 2001, adroitly elaborates on this point in his attempt to distinguish the varieties of function endorsed within osteopathy as distinct from the part-functionalism predominantly embraced by the medical community). 
Although there are a number of objections to SF (Ananth 2017; Perlman 2010), it is worth noting that it says very little about biological features as evolved features the same omission in most PHJs. Given this omission, the pressing objection from an EM perspective is that $\mathbf{S F}$ is unable to distinguish genuine functions from accidental side effects. For instance, the heart's thumping sound is a capacity of the heart, but the system to which the heart contributes, namely, the circulatory system, performs the task or has the capacity for blood circulation. The heartthumping sound does not contribute to this overall performance, so this capacity should not be considered a function. Yet, the systemic functionalists appear to be forced into accepting the thumping sound as a genuine function of the heart.

Additionally, and more pressing to the discussion at hand, the part-functionalism that underwrites $\mathbf{S F}$ obscures a proper understanding of the body as an integrated evolved entity. This includes understanding the human body as an evolved ecologically oriented entity, something not well integrated into current PHJs. Depew (1998, p. 31) expresses this point when he reminds Darwinians that biological systems qua bodies are "bounded, informed, autocatalytic dissipative systems [and] are by definition parts of ecological communities, and that the information which they store and use is subject to dynamics that are inseparably both competitive and cooperative... For natural selection can play the deep, essential, and above all creative roles suggested by their theories only when organisms are treated ecologically." The upshot is that the field of medicine should be cautious about incorporating solely $\mathbf{S F}$ into its understanding of the human body.

\section{Evolutionary Functionalism}

In an attempt to distinguish genuine functions from accidental side effects and be more sensitive to the sense of body qua ecological entity suggested by Depew above, Darwinians have insisted that an evolutionary selectionist account must be incorporated into the concept of function. Drawing from the work of Mayr (1974) and Wright (1976), Evolutionary Functionalism (hereafter EF) developed as follows:

Evolutionary Functionalism: A feature $\mathbf{X}$ has a function in an organism $\mathbf{O}$ if and only if activity $\mathbf{Y}$ of $\mathbf{X}$ produces effect $\mathbf{E}$ because $\mathbf{Y}$ and $\mathbf{E}$ were naturally selected (over some other causes and effects) to bring about the goals $\mathbf{G}$ of survival and reproductive success of $\mathbf{O}$.

For example, the liver has a function of blood detoxification in mammals, because the activity of converting ammonia into the less toxic compound urea produces the effect of detoxified blood. Moreover, this activity and effect were naturally selected for the sake of survival and reproduction. It is this selectionist account that allows for distinguishing genuine functions from side effects; that is, the thumping sound of the heart is a side effect of the naturally selected effect of blood pumping, or the far less noticeable sounds of detoxified blood moving out of the liver are side effects of the naturally selected effect of blood detoxification. 


\section{Reply to Evolutionary Functionalism}

At first glance, EF as an alternative or supplement to $\mathbf{S F}$ seems plausible because it is able to distinguish genuine functions from side effects and appears to be sensitive to the body as an evolved ecologically based unit, but it has had its detractors within the Darwinian camp. Specifically, there are more subtleties to EF than are captured in the above definition. For instance, how to understand "selection" in the above definition is not made clear. Are features currently selected? Were they only selected in the past? Will they be selected in the future? Is it really possible to make sense of health and disease by way of EF? These questions suggest that greater specificity is required in order to put forth an evolutionary concept of function that is a worthy alternative to SF. Specifically, three philosophical approaches to understanding EF as they pertain to health and disease - will be distinguished: (i) the backward-looking Etiological Evolutionary Functionalism and (ii) the forward-looking Propensity Evolutionary Functionalism. Additionally, this section will argue that neither of these versions of biological function is adequate, but that a worthy alternative, (iii) Mixed Evolutionary Functionalism, is available.

\section{Etiological Evolutionary Functionalism}

According to Etiological Evolutionary Functionalism (hereafter EEF], a feature performs a function in a system of an organism if and only if (1) the feature's presence in a system was useful with respect to the organism's reproductive success in previous generations and that (2) it is the result of evolutionary selection forces. From this perspective, a trait is functional because its presence is due to its ability to produce, in a self-sustaining fashion, a beneficial difference that related traits were unable to produce. The result is that, in contrast to side effects or lucky features, what counts as a functional trait is one that can recycle itself as a result of delivering $a$ reproductive advantage to the organisms of which it is a part (Millikan 1993, p. 38 and Hardcastle 1999, p. 32; my italics). The formal definition of EEF looks like this:

Etiological Evolutionary Functionalism: A feature $\mathbf{X}$ currently has a function in an organism $\mathbf{O}$ if and only if activity $\mathbf{Y}$ of $\mathbf{X}$ produces effect $\mathbf{E}$ because $\mathbf{Y}$ and $\mathbf{E}$ were naturally selected (over some other causes and effects) to bring about the goals $\mathbf{G}$ of survival and reproductive success of $\mathbf{O}$.

With this general description in place, a specific example is in order. In humans, iduronate sulfatase is the lysosomal enzyme that is designed to breakdown mucopolysaccharides (a gel-like substance found in the body of cells). For example, connective tissue outside of cells needs to be replaced on occasion. When this replacement occurs, iduronate sulfatase metabolizes the old connective tissue. On occasion, in males only, a genetic error occurs such that not enough iduronate sulfatase is present to break down the mucopolysaccharides that build up from the remaining old connective tissue. The result of the buildup of mucopolysaccharides (in lysosome cells) is the following multisystem collapse: hyperactivity, aggressive behavior, coarse facial features, enlargement of internal organs, dwarfism, stiffening 
of joints, progressive deafness, and severe mental retardation. This genetic disease is known as Hunter syndrome.

From the EEF perspective, the function of these iduronate sulfatase enzymes is to metabolize mucopolysaccharides, because, ancestrally, there was selection pressure in favor of them doing just this to ensure survival and reproductive success. Notice that this understanding of iduronate sulfatase enzymes not only makes sense of the specific function of metabolizing mucopolysaccharides but also gives consideration to the organism as a whole in terms of survival and reproductive success. Additionally, how well iduronate sulfatase enzymes function is a product of how these enzymes function in relation to other neighboring organelles in the cell; its evolved functions are understood as adaptive compromises with respect to other related organelle functions. In severe cases, human males who either lack iduronate sulfatase enzymes or do not produce enough of them have multisystem dysfunction, rendering them physically unfit and thus unhealthy (see Bechtel 1985 for further discussion). Again, such fitness assessments can complement PHJs when thinking about the body as an evolving ecologically conceived bundle of compromises.

\section{Replies to Etiological Evolutionary Functionalism}

A quick glance at $\mathbf{E E F}$ might move one to consider this version of $\mathbf{E F}$ credible. For, as part of its content, it appears to include both the necessary and sufficient conditions for what it means for $\mathbf{X}$ to have a function and is respectful of the body as an evolving entity. As Bigelow and Pargetter affirm, "The big plus for the etiological theory is that it makes biological functions genuinely explanatory, and explanatory in a way most comfortable with the modern biological sciences" (Bigelow and Pargetter 1987, p. 187). Moreover, it provides a general framework for distinguishing genuine functions from mere accidents, because a feature of a biological system is a function if and only if it is the product of natural selection.

By relying solely on evolution, however, EEF must ascribe functions to those features that no longer have functions. As Nissen correctly remarks, "Since history is forever, if functions are determined by their history, functions are forever. New functions can be added, but old ones never die. This means that vestigial organs still have their original functions" (Nissen 1997, p. 185). Thus, EEF is triumphant in distinguishing genuine functions from accidents because of its reliance on evolutionary causal history, but such an achievement proves to be a somewhat pyrrhic victory in that vestigial organs, like the appendix, human tailbone, and human male nipples, do not lose their evolved functions - even if those functions can no longer be actualized.

There is one additional concern that needs to be noted. Some argue that EEF is committed to defining functions in terms of actual reproductive success. As it is described in this section, it is ambiguous whether or not such a commitment is entailed. Still, Bigelow and Pargetter note that some scholars argue that EEF assumes that 
fitness can be judged only retrospectively: that it is only after we have seen which creatures survived that we can judge which were the fittest; moreover, it assumes that the fact that certain creatures have survived, whereas others did not, is what constitutes their being fit. (Bigelow and Pargetter 1987, p. 190)

Clearly, this may be a serious problem with versions of EEF that are committed to the view that $\mathbf{Y}$ is a function of $\mathbf{X}$ if and only if $\mathbf{Y}$ contributes to the actual reproductive success of $\mathbf{O}$. The concern is that it is absurd to confer a function on an organism or take away a function from an organism based on lucky or unlucky anomalous environmental perturbations, which are not part of the normal environment in which the organism has evolved.

It is this sort of worry that leads Bigelow and Pargetter to claim that the "etiological theory is mistaken in defining functions purely retrospectively, in terms of actual survival" (Bigelow and Pargetter 1987, p. 191). So, if it is the case that EEF is committed to actual reproductive success, then this criticism is quite relevant and should influence PHJs accordingly; that is, for all of its pluses, EEF should not be solely employed as the sense of function anchoring the concept of biological function for PHJs.

\section{Propensity Evolutionary Functionalism}

The point that can be taken away from the above criticism is that it is better to say that $\mathbf{Y}$ is a function of $\mathbf{X}$ if and only if $\mathbf{Y}$ has the capacity its ancestors had to contribute to the reproductive success of $\mathbf{O}$. By substituting "capacity" for "actuality," the problem of environmental anomalies disappears. This capacity view of biological function is known as the propensity interpretation. For example, the function of iduronate sulfatase enzymes is to metabolize mucopolysaccharides and not some other substance found in the body of cells, because creatures whose mucopolysaccharides are broken down by iduronate sulfatase enzymes have a greater disposition of surviving and reproducing than creatures whose mucopolysaccharides cannot be metabolized. Propensity Evolutionary Functionalism (hereafter PEF) can be defined as follows:

Propensity Evolutionary Functionalism: A feature $\mathbf{X}$ has a function in an organism $\mathbf{O}$ by performing activity $\mathbf{Y}$ if and only if $\mathbf{Y}$ produces effect $\mathbf{E}$ because $\mathbf{Y}$ and $\mathbf{E}$ confer and will continue to confer a propensity $\mathbf{P}$ (within a certain range of environmental pressures) to bring about the goals $\mathbf{G}$ of survival and reproductive success of $\mathbf{O}$.

In thinking about a healthy biological system, Bechtel endorses the analysis offered by Bigelow and Pargetter as follows:

There is [a] conceptually intermediate position that has been developed in philosophical reflections on evolutionary theory - a propensity interpretation of fitness. What the propensity interpretation of fitness does is define fitness in terms of propensity to reproduce, not reproductive success itself. This is all that is required for our purposes, for we can now define something as functional if it increases the propensity of its bearer to reproduce ....(Bechtel 
1985, p. 151; my italics). The approach I am exploring directs one to engage in an engineering analysis to identify how the physiological organization of the system equips it to deal with the selection forces working upon it. A healthy state of the system is one in which it makes best use of its physiological endowments in responding to selection pressures. (Bechtel 1985, p. 154)

Bechtel argues that the concept of function that should be relevant to the concept of a healthy state of a system is PEF. From this perspective, PHJs can endorse a notion of a healthy body by understanding how features contribute to the propensity or likelihood that said body will survive and reproduce.

\section{Replies to Propensity Evolutionary Functionalism}

The difficulty with PEF is that it takes function as ontologically prior to selection - a move that begs the question of how PEF is able to determine what is and is not a function. As Bigelow and Pargetter note (1987, p. 192; my italics), "On our theory, the character already has the function, and by bad luck it might not survive, but with luck it may survive, and it may survive because it has a function." Similarly, in response to the etiological account, Bechtel claims (1985, p. 150; my italics), "The correct order is to claim that those things that are functional will evolve, rather than to claim that those things that evolve are functional."

The above claims by Bigelow, Pargetter, and Bechtel are problematic. The obvious problem with giving priority to function over selection is that it begs the question of why it is the case that $\mathbf{X}$ has the function in the first place. $\mathbf{X}$ has the function to do $\mathbf{Y}$, because $\mathbf{X}$ yields a survival-enhancing propensity on $\mathbf{O}$. But why does $\mathbf{X}$ have the function qua survival-enhancing propensity that it has? Clearly, they cannot rely on propensity here, because propensity and function are one and the same once selection is no longer part of the concept of function. That is, if Bigelow, Pargetter, and Bechtel presume (as they do) that character $\mathbf{X}$ "already has the function" prior to selection, then this means that $\mathbf{X}$ already has a propensity prior to selection. If not selection, then what confers "having a propensity" that makes it the case that $\mathbf{X}$ is a function? They could respond by claiming that a propensity is a property or capacity of a trait to do $\mathbf{X}$. Yet, this leads to a regress problem. For now it can be asked, how is it the case that a property or capacity "already" exists in a creature without introducing some sort of causal history to account for the capacity? As it stands, Bigelow, Pargetter, and Bechtel have no answer, because a capacity or property is an unexplained metaphysical element of their analyses. Natural selection, on the other hand, is a physical force or process (like gravity). Are propensities thought to be the same? This seems unlikely, because Bigelow and Pargetter have already ruled out the possibility that propensity relies on contingent natural phenomena. The upshot of this overall objection is that Bigelow, Pargetter, and Bechtel have not offered a persuasive account of what a function is. Thus, it is not at all clear that giving priority to propensity over selection is preferable. 
Bigelow, Pargetter, and Bechtel might object to the above criticism by claiming that "What is a function?" is different from the question "What causes functions to be present?" For example, if Bigelow and Pargetter find a watch, they can claim to know it's a watch first and then ask who made it. Obviously, they know that they have to give a causal history to account for its function, but that is different from defining what a function is. That is, they could argue that they do not have to answer the second question in order to answer the first. This reply, however, is vulnerable to the next criticism.

Setting aside the ontological priority worry, it is not clear that the PEF can distinguish genuine functions from mere side effects. For example, imagine that the metabolic activities of iduronate sulfatase enzymes not only break down mucopolysaccharides, but they also have the accidental benefit of improving the sense of smell. On the propensity interpretation, both the metabolic activities and the improved sense of smell would have to be considered genuine functions because the former (directly) confers a survival-enhancing propensity and the latter (accidentally) confers a survival-enhancing propensity. Peter McLaughlin voices a somewhat similar concern as follows:

'The function of $[\mathbf{X}]$ is $\mathbf{Y}$ ' is true, not only when $\mathbf{X}$ does $\mathbf{Y}$ due to its propensity, but also when it has a strong propensity to do $\mathbf{Y}$, but happens to do it by accident and not due to its propensity... Furthermore, if low probabilities were to count as low propensities, then it would seem that even accidents occur on account of a propensity. (McLaughlin 2001, p. 126)

The implication of the above account leads McLaughlin to conclude correctly that those who embrace the history-free propensity interpretation of fitness are "forced to attribute a function to more or less everything" (McLaughlin 2001, p. 126). Indeed, this criticism reveals why they must address the question "What causes functions to be present?" Thus, the PEF should be rejected by medical professionals as being an integral part of the concept of a healthy evolving body on the grounds that it not only gives ontological priority of place to function over natural selection but also because it cannot distinguish genuine functions from mere side effects.

\section{Mixed Evolutionary Functionalism}

Thus far, the general conclusion is that EF, EEF, and PEF are not able to emerge unscathed in route to their respective concept of biological function. EEF suffers from focusing on actual reproductive success and not being able to allow an entity to lose its function, whereas PEF cannot distinguish genuine functions from fortuitous accidents and it cannot justify giving priority to function over selection.

The more defensible alternative combines these three accounts. PEF has the advantage that it is not committed to the actual reproductive success of a trait, but only to the disposition of such a trait to enhance reproductive success. The advantage of both $\mathbf{E F}$ and EEF is that they are able to distinguish genuine functions from mere side effects. Moreover, EEF gives priority to selection over propensity in order to 
determine what is a genuine function because it takes causal history into account. In the spirit of unification, the appropriate account of function will give priority to natural selection, but claim that selection ranges over propensities to survive and reproduce. In full, a feature of an organism is a function if and only if it confers a propensity to produce a specific set of activities/effects and corresponding specific benefits and to enhance the goal of survival and reproductive success on an organism and that such a propensity set is established through natural selection. The formal characterization of this dual-functional mixed account (hereafter MEF) is as follows:

Mixed Evolutionary Functionalism: A kind of EF explanation maintains that a feature $\mathbf{X}$ has a function in an organism $\mathbf{O}$ by performing an activity $\mathbf{Y}$ if and only if $\mathbf{Y}$ produces effect $\mathbf{E}$, and both $\mathbf{Y}$ and $\mathbf{E}$ confer a survival-enhancing propensity $\mathbf{P}$ on $\mathbf{O}$ (within a certain range of environmental pressures) and will continue to confer $\mathbf{P}$ on $\mathbf{O}$ (so long as a certain range of environmental pressures is present). And, moreover, $\mathbf{P}$ is currently present, because, ancestrally, there was natural selection in favor of retaining $\mathbf{P}$ to bring about the goals $\mathbf{G}$ of survival and reproduction.

A return to the enzyme example will help to explain the above account. Recall that iduronate sulfatase is the lysosomal enzyme that is designed to break down mucopolysaccharides. With respect to the mixed account above, iduronate sulfatase is a function of the human organism, because of its ability to produce the specific effect/activity of metabolizing mucopolysaccharides; and this effect correspondingly confers a survival-enhancing propensity on the human organism. Importantly, the reason why it currently confers such a propensity is because, ancestrally, there was natural selection in favor of retaining such a propensity (over a range of environmental pressures) for the sake of survival and reproduction (Ananth 2008).

\section{Benefits to Medicine}

Early in this chapter, it was stressed that the body as a bundle of evolutionary compromises captures the principal sense of how the body should be understood within the framework of EM. Two key aspects of understanding the body in this way is that "the bundle" must be understood in terms of proper functioning and homeostasis. Although medicine currently presumes almost exclusively something like $\mathbf{S F}$, this analysis has suggested that $\mathbf{E F}$ should supplement or complement it. Specifically, MEF is the version of EF that seems most reasonable. What this means is that the evolutionary compromises of the body should be understood as compromises that manifest their activities as suggested by MEF. So, if it is correct that $\mathbf{S F}$ is an integral part of how medicine understands the human body, then MEF should correspondingly be included in this understanding. 


\section{Evolutionary Dual Homeostasis}

\section{Intercellular Homeostasis}

If it is acknowledged that the body is best understood as an evolutionary functioning bundle of compromises, then it should be no surprise that there is an intricate balancing act that has unfolded within the body (Bernard 1957/1865 and Cannon 1963) and between the body and its environment (Goldstein 1963 and Depew 1998). Moving beyond the efforts of Bernard and Cannon (Cooper 2008), the research regarding bodily homeostasis continues to this day on both the biomedical front and the philosophical head. For instance, on the biology side, Keesey and Powley (2008, p. 445; my italics) have stressed that "though the early workers failed to include body energy among the conditions of the body subject to homeostatic regulation, a sound foundation, based upon the work of the past several decades, appears now to be in place for its inclusion." Additionally, on the philosophical flank, as part of his defense of a naturalistic concept of health, Boorse (1997, pp. 78-79; bracketed addition mine) acknowledges that his account assumes homeostasis as a necessary condition. He elaborates as follows:

\footnotetext{
Though I did not stress the dynamism [i.e., the process of homeostasis] of normal physiology in presenting [my naturalistic concept of health], I always assumed it ... Obviously, no fact is more pervasive than what is often called 'dynamic equilibrium' of normal physiology: the normal functional variation within organisms acting and reacting to their environment. The normal level of almost all part-functions varies with what an organism is doing, what other part-functions are being performed, and the environment ... A common pattern is that environmental stress evokes short-term compensatory functions that maintain homeostasis up to a point, but beyond that point the coping mechanisms break down and a discontinuity, a discrete state of illness, results.
}

It is now appropriate to sketch the framework of a homeostatic system. The general idea is that an individual organism's internal environment is in homeostasis if it responds appropriately to various stimuli from the internal and external environment. Specifically, on the physiological level, the following internal physical states must be kept stable for the intercellular fluid to be in homeostasis (Roberts 1986, p. 201):

(i) The chemical composition of the intercellular fluid (e.g., constant level of glucose in the bloodstream)

(ii) The osmotic pressure of the intercellular fluid (determined by the relative amounts of water and solutes)

(iii) The level of carbon dioxide in the intercellular fluid

(iv) The temperature of the intercellular fluid

(v) The elimination of waste from the intercellular fluid

If the above five states of the intercellular fluid are held fairly constant, then the internal environment is considered to be in homeostasis. Recall that homeostasis is maintained by both positive and negative feedback. Many organs and organ systems 


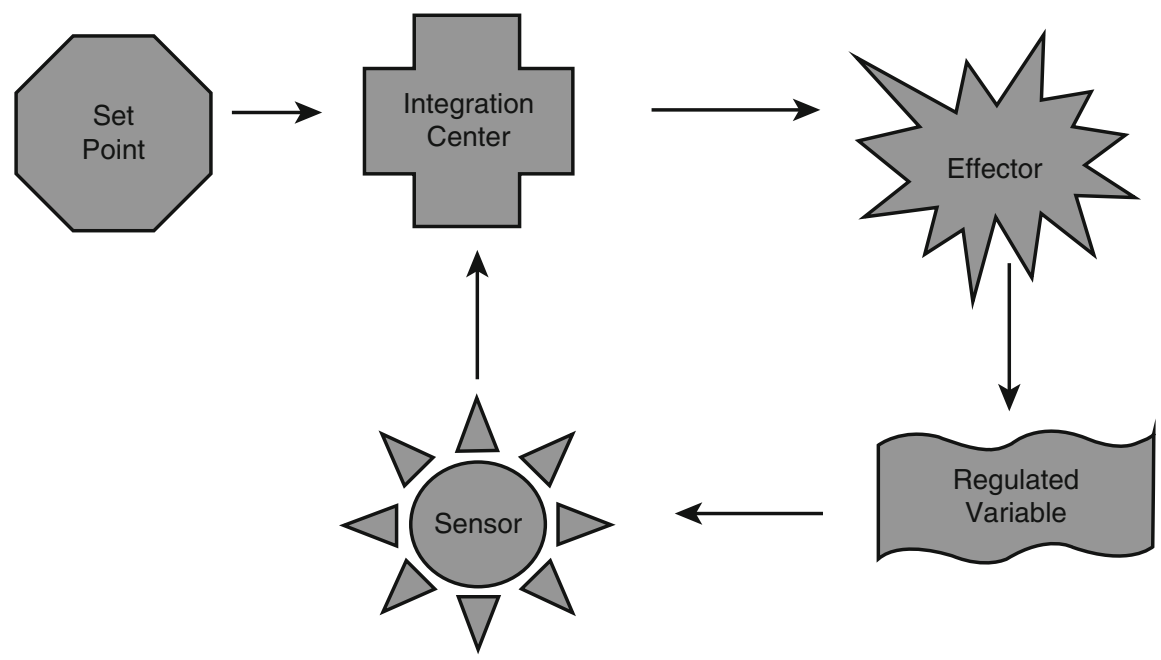

Fig. 1 Standard homeostatic system (With permission from McGraw-Hill Education)

of the body are designed by natural selection to secure intercellular homeostasis (Basanta, et al. 2008). For example, it is crucial that intercellular temperature be within a certain range so that metabolic processes can occur. To this end, overall body temperature must remain at a certain level to ensure that intercellular homeostasis is maintained. This example is a glimpse into the interconnected and hierarchical nature of the human body. As a way of elaborating on the discussion, Fig. 1 from Seidel (2002, p. 3) offers a pictorial look at the five elements of a standard homeostatic system.

Through the example of body temperature, the above five components can be summarized as follows (Roberts 1986, pp. 205-10):

(i) Regulated variable is a variable that is kept constant. For example, the following are regulated variables: body temperature, blood pressure, and the blood content of glucose, oxygen, and potassium ions. (Note that heart rate, cardiac resistance, urine output, and breathing rate are not regulated outputs. Rather, they are usually understood as effectors, which are designed to maintain set point levels.)

(ii) Set point is a quantitative value for the regulated variable. For example, $98^{\circ} \mathrm{F}$ is the approximate temperature of the interior of the human body.

(iii) Sensor(s) assesses the current status of the regulated variable. The anterior hypothalamus and the skin are the temperature-sensor organs of the human body.

(iv) Integration center compares current conditions with the set point. The anterior hypothalamus is the organ that acts as the integration center for the human body. It receives the information about surface body temperature from skin nerve endings. It compares this information with the set point. 
(v) Effector brings current status of the regulated variable into line with the set point. With respect to body temperature, this feedback process is also initiated by the hypothalamus and is an effector along with the anterior and posterior hypothalamus. If the body temperature is above the set point (i.e., overheated), then sweat production is initiated, and the shivering center is inhibited in order to return the body's temperature to its set point. If the body temperature is below the set point (i.e., under-heated), then cellular metabolism is increased through the anterior hypothalamus, and shivering is increased through the posterior hypothalamus in order to return the body's temperature to its set point. If there is a foreign invader (e.g., bacteria), body temperature can rise in an attempt to destroy it. In the case of a fever, the set point itself increases as well.

The above elements of homeostasis are relevant to the various organs and organ systems of the body. The result is a feedback loop between the intercellular fluid and many of the other structures of the body.

\section{Organism Homeostasis}

What about the physiological functions of the body as a whole? Boorse hints at this concern in the above quotation when he defines dynamic equilibrium as "the normal functional variation within organisms acting and reacting to their environment." Then he goes on to claim that "the normal level of all part-functions varies with what the organism is doing" (my italics). Boorse's use of "within organism" suggests that he is concerned with how the internal part-functions of organisms react to their environment, but his use of "what the organism is doing" suggests that internal partfunctions maintain their dynamic equilibrium qua homeostasis with respect to the physiological activities of the organism. For example, eating, waste removal, sleeping, running, walking, etc. are functions of the body (not merely any particular part) as a whole that help to sustain intercellular fluid. Where this analysis departs from Boorse is that intercellular homeostasis, which is associated with the integrated internal activities of the body, is distinct from the external behaviors of the body. These external behaviors are distinct evolved patterns that are not only being influenced by intercellular fluid, but are also influencing intercellular homeostasis (Ananth 2008). Therefore, the idea is that there is a dual-homeostatic interaction between behavioral activities of organisms as a whole and their intercellular activities.

For example, sleeping, eating, and waste removal are necessary for regaining lost energy. Energy restoration is crucial not only for carrying out intercellular processes but also for the organism as it contends with daily environmental disturbances. Importantly, these physiological activities are coordinated activities of the organism as a whole as it interacts with its environment - they cannot be understood in terms of parts alone. What part of the body pumps blood? What part of the body has the function of walking? What part of the body has the function of sleeping? The answer 
to the first question is the heart. The remaining questions do not have such a straightforward answer. The reason is that walking, sleeping, eating, reproducing, swimming, etc. are evolved coordinated activities of an organism as a whole in relation to its environment. It is the body as a whole that walks. In general, the legs, the arms, and torso coordinate to create a pattern of activity called walking. Similarly, it is the body as a whole that reproduces. It is the body as a whole that sleeps. It is these sorts of physical activities of the whole body that cannot be captured by only a part-functionalism or intercellular homeostasis, but is relevant to what an organism is doing as a whole entity. It is to these sorts of activities that "organism homeostasis" refers.

In defense of this holistic notion of body movement, Goldstein (1963, pp. 229-230) says that when humans make a certain movement

we do not innervate individual muscles or muscle groups, but a change in the present state of innervation of all the body muscles takes place. Thus, a pattern of innervation results, in which one definite single contraction, namely, the one which is intended, stands in the foreground. For the appropriate contraction of one muscle group, i.e. for that contraction by which a definite effect results, a certain state of innervation of the remaining body muscles is requisite. To be sure, we do not notice this state of innervation, because it seems to be insignificant for the intention of that movement. But it is not at all insignificant, it rather enables the organism to execute the movement correctly.

As Goldstein makes clear, specific body movements require that all (he probably means most) body muscles (in addition to the specific muscles of a particular movement) be coordinated or stimulated to ensure that the specific body movement is accomplished. The pattern of movement that emerges is in conjunction with the pressures from the external environment. Drawing from Goldstein's account, it is this pattern of movement in response to environmental stress that is the product of natural selection. For example, the overall patterns of swimming motion of fishes or flying patterns in birds are evolved patterns that are crucial to survival and reproductive success. It is these sorts of behavioral patterns that allow organisms to interact in an energetically balanced way with their environments. It is the energy balance created by these coordinated behaviors that is here being called organism homeostasis. On this view, organisms share a close relationship with their environment such that energy balance is part of understanding organisms as ecologically oriented creatures (Depew 1998 and Keesey and Powley 2008).

Thus, along with intercellular homeostasis, organism homeostasis must be included in the discussion of homeostasis. That is, since it is the overall organism that directly contends with the environment, intercellular homeostasis can be viewed as a necessary condition for overall physiological homeostasis, which is crucial to both intercellular homeostasis and survival and reproduction. For instance, walking, running, sleeping, jumping, grasping, and other evolved behavioral activities can be viewed as effectors that are crucial to maintaining an organism's life (i.e., energy balance maintenance) under a certain range of environmental influences. 
In his defense of a naturalistic concept of health, Bechtel $(1985$, p. 149) hints at this sense of "organism homeostasis":

\begin{abstract}
The idea that living organisms incorporate a complex organization that makes them homeostatic systems provides an important element needed in a satisfactory physiological concept of health. In terms of it, one can define a healthy system as one that is at or near its designed equilibrium state. Significant deviations, especially those in which some external agency is required to restore the system to the equilibrium state, are disease states.
\end{abstract}

According to Bechtel, the complexity of the human body is sufficient to understand it as a homeostatic system. Notice that this claim is distinct from the idea that the human body is composed of homeostatic systems. The first claim refers to what is here being called organism homeostasis, while the second claim refers to internal homeostasis. To this end, the physical activities of the brain (or specific parts) can be seen as the integration centers that assist in fight-flight responses, resting responses, bathing responses, etc. These overall physical functions of the body are not easily captured by a strict part-functionalist account. Rather, this requirement of dual homeostasis reveals that there are functions that can be attributed to the organism as a whole. The upshot is that, once the discussion on health includes homeostasis, organic functional holism is compatible with part-functionalism.

This section has offered a brief glimpse into the dual nature of the concept of homeostasis with respect to the evolved body. It included a discussion of both intercellular homeostasis and organism homeostasis and a general explanation of the different elements that comprise a homeostatic system. The general conclusion that should be drawn from this section is that homeostasis should include not only the internal intercellular balances of the body, but the many behavioral activities of the body designed to contend directly with the environment.

\title{
Bringing the Bundle Together
}

This chapter began with a plea from the EM camp that medical practitioners and educators ought to incorporate evolutionary thinking into both their PHJs and overall pedagogy. This plea requires accepting a set of philosophical and physical concepts that make adequate sense of the body as an evolving system. Endorsing and implementing such a request also require that the body be taken seriously as a bundle of evolutionary compromises. Moving in this direction further requires endorsing a specific concept of biological function, namely, MEF. Additionally, the body as a dual-homeostatic system completes such an account of the body as an ecologically bound system. Synthesizing all this reveals that, from the perspective of EM, the body is best understood as an ecologically oriented and evolutionary dynamic dual homeostatic properly functioning holistic system. 


\section{The Evolved Bundled-Homeostatic Body: Influence on the Practice of Medicine and Public Health}

Given the predominantly theoretical analyses of the previous sections, it is reasonable to offer a number of examples that reveal the usefulness to medical practitioners of including an EM perspective in their conceptual tool kit. The examples included below are (1) defense mechanism of fever, (2) genetic disease of sickle-cell anemia, and (3) the immunological response of allergies.

\section{Defense Mechanism: The Fever}

The body has many defense mechanisms of which fever is a classic example. In general, a fever is indicative of the body attacking a pathogen. In mammals, the internal heat created by fever is an evolved immunological feature of the body that has the function (i.e., MEF) to destroy and/or mitigate the proliferation of a foreign invader. All of this is clear to the medical community, so what can EM contribute to this case? For a start, it is important to keep in mind that pathogens need to be understood in terms of a pathogen-host-environment relationship. As noted before, since adaptations are adaptations to local environments, it is eminently reasonable that there are pathogens that cannot be controlled by way of a febrile response because the class of pathogen has either evolved to withstand the fever response or it is a species of pathogen that is foreign to the range of environmental dynamism with which the human body has dealt. This means at least one of two things. One, pathogens are able to produce variations (as a result of their rapid reproductive rates) that can withstand the febrile defense response - human evolutionary adaptations cannot keep pace with the swift evolutionary changes exhibited by some kinds of rapid reproducing organisms. Two, the foreign pathogen is a novel invader that is either unencumbered by the febrile response or benefits from such a pyretic environment. Although all of these possibilities could be hypothesized without necessarily employing the tenets of $\mathbf{E M}$, an evolutionary take could very well assist in rendering the realities of such possibilities, if missed from the traditional partfunctionalist/mechanical medical model, and could guide future experiments regarding the presence of these sorts of unanticipated variations.

Indeed, artificial attempts to control a fever, either by allowing it to persist or stopping it (e.g., the use of aspirin or acetaminophen), run the risk of controlling certain bacteria while simultaneously allowing for the proliferation of other viruses. For example, not lowering a fever can reduce the availability of iron to the body and this can mitigate the growth of some bacteria. (Again, this should not be a surprise when thinking of the body as a bundle of compromises in the attempt to maintain energy homeostasis - heat increase compromises quantity of iron in the body.) Simultaneously, this reduction in iron can cause, for example, E. coli and Vibrio cholera to produce toxins that actually exacerbate illnesses. In contrast, there is some 
evidence that artificially hindering a chicken pox-induced fever in children resulted in slower recovery than those who only took a placebo. Also, in another study, patients who took aspirin or acetaminophen for a common cold had a poorer antibody response and greater nasal stuffiness than those who were on a placebo (Nesse and Williams 1994, pp. 27-28). As Nesse and Williams stress (1994, p. 29), "The important point, with respect to the adaptive significance of fever, is that we need to know what we are doing before we interfere with it... We hope that medical research will soon produce the evidence to help doctors and patients decide when fever is and is not useful." Yet, this should not be perceived as a trivial task given the nature of the human body. As Ewald (1994, p. 19) cautiously points out, "fever could be a weapon that backfires, causing worse disease than would be present without fever." Thus, EM proponents are stressing that distinguishing defenses from other expressions of infection requires in part to "respect the evolved wisdom of the body" (Williams and Nesse 1994, p. 31).

This is a reminder that the human body as an ecologically oriented bundle of evolutionary compromises must contend with other organisms that have their unique compromised bundles that are subject to selection. For example, researchers (Bishai et al. 1996) treated a patient with rifampin (an antibiotic) in an attempt to destroy the tuberculosis bacteria, and it appeared that this antibiotic was effective; indeed, doctors could not culture any tuberculosis bacteria in the lungs of the patient to whom rifampin was given. Unfortunately, the patient did succumb, and upon autopsy and DNA sequencing, it was determined that a mutated version of the tuberculosis bacteria was able to withstand the rifampin. As Freeman and Herron (2004, p. 511) point out, "the data are consistent with the hypothesis that bacterial populations evolve in response to selection imposed by antibiotics." So, from an EM perspective, this sort of example reveals that not only is the human body a bundle of evolutionary compromises, but that there can be organisms and cells within the body that are themselves evolving in response to human intervention. At the very least, the EM perspective can be employed in supplementing the view that the judicious use of antibiotics is paramount when bundles of evolutionary compromises are competing with and within one another.

Additionally, the use of aspirin suppresses fever, pain, and inflammation, while acetaminophen suppresses only fever and pain. This is important because inflammation can reduce the proliferation of some viruses by allowing additional defense responses at the point of inflammation and reducing the flow of blood and thus movement of the viruses from the infection area (Ewald 1994, p. 21). For instance, Ewald (1994, p. 22) tells us that certain infections in mice are exacerbated as a result of inflammation suppression via anti-inflammatory drugs. What this means is that an EM perspective regarding variation in viruses could determine whether or not to use aspirin or acetaminophen to treat a particular illness and to be on the lookout for such variations. Drawing upon similar EM insights with respect to cholera and dysentery, it is clear why Ewald (1994, p. 19) tenders the following conclusion: "Evolutionary and biochemical principles therefore suggest that the overall net effect of fever may be positive or negative... Because these alternative evolutionary scenarios have not been generally recognized, key experiments to distinguish between them have not 
been done." Thus, the fever example illustrates how EM can supplement the efforts and directions of biomedical research as well as decisions made by doctors caring for patients.

\section{Genetic Disease and PHJs: Sickle-Cell Anemia}

Sickle-cell anemia is a blood disorder caused by a gene that is also beneficial. This disorder occurs mostly in people from Africa (and some parts of India), where malaria is a major cause of death. To understand sickle-cell anemia, a few definitions related to genetics need to be made clear. First, alleles are alternative forms of a particular gene that affect a specific trait in different ways. For example, consider eye color. Assume that brown eyes are "dominant" over blue eyes. "B" refers to a dominant allele. "b" refers to a recessive allele. The gene for brown-colored eyes includes the following set of alleles: $\mathbf{B B}$ and $\mathbf{B b}$. The alleles for blue eyes are $\mathbf{b b}$. $\mathbf{B B}$ is a condition known as homozygous dominant. This means that so long as BB alleles are present, brown-colored eyes will always be present over any other colored eyes. Bb is the condition known as heterozygous. In this case, a person has both a dominant and a recessive gene. In heterozygous cases, the dominant allele swamps the effects of the recessive allele. So, Bb will produce brown eyes, even though a recessive gene is present. Finally, bb refers to the condition known as homozygous recessive. In this case, a person has two recessive alleles. With respect to eye color, bb will produce blue eyes.

In principle, sickle-cell anemia occurs in a similar way, but the effect under consideration is red blood cell modification with respect to malaria parasites. Assume that $\mathbf{R R}$ is the homozygous dominant condition, $\mathbf{R r}$ is the heterozygous condition, and $\mathbf{r r}$ is the homozygous recessive condition. Genetically, in a simplified rendering, the three conditions produce the following effects:

1. Homozygous Dominant: These people carry two of the same forms of the gene (alleles) and are not able to modify the shape of their red blood cells. Although there are no detrimental side effects, these people are unable to defend against malaria parasites.

2. Homozygous Recessive: These people carry two recessive alleles that are able to modify the shape of their red blood cells. However, as result of this modification, they also suffer crippling side effects. This group of people is said to have sicklecell disease.

3. Heterozygous: These people carry one dominant and one recessive allele. The recessive gene $\mathbf{r}$ is able to modify the shape of the red blood cells. Moreover, the combination of $\mathbf{R r}$ defends against malaria without any serious crippling effects in certain environments.

Nesse and Williams (1994, p. 99) report on people with the homozygous recessive condition as follows: "Their red blood cells twist into a crescent or sickle shape that cannot circulate normally, thus causing bleeding, shortness of breath, and pain in bones, 
muscles, and the abdomen." Again, these people are said to have the sickle-cell disease. Those who are homozygous dominant for this gene have normal red blood corpuscles, but are unable to defend themselves against malaria. However, those who are heterozygous for the gene have the sickle-cell trait. These people have a hemoglobin structure that is able to remove the infected malaria parasites before they cause serious damage to the body (for further details, see Salthe 1998, p. 15 and Kark 2000, url). Much like Down's syndrome, sickle-cell anemia (the homozygous recessive condition) is a genetic disease and can only be acquired through the genes of parents.

How are we to make sense of PHJs with respect to sickle-cell anemia? From the EM perspective, with respect to those who have the homozygous recessive genes, they are deemed unhealthy (Ananth 2008). Both intercellular fluid and organism homeostasis are greatly disrupted, rendering these people very unhealthy. In contrast, people who are homozygous dominant become unhealthy only if they contract malaria. In the case of those who are homozygous dominant, it is clear that the role of the environment is crucial to their health. So long as these people do not live in malaria-infested areas, they will have no health concerns with respect to their genetic condition. In contrast, the homozygous recessive condition will render a person very unhealthy in just about any environmental condition, because the deformation of the hemoglobin is an inevitable consequence of being homozygous recessive.

Now, the heterozygous condition must be assessed. At first glance, it appears that this condition is healthy, since malaria can be destroyed. From an EM perspective, Nesse and Williams (1994, p. 99; bracketed additions mine) offer the following summary judgment:

The sickle-cell gene thus illustrates heterozygote advantage. Because of their resistance to malaria, heterozygotes are favored over both kinds of homozygotes: Homozygotes [who are recessive] for the sickle-cell allele have low fitness resulting from sickle-cell disease, while homozygotes [who are dominant] for the normal allele have low fitness resulting from their vulnerability to malaria.

One additional point needs to be made explicit concerning the above description. Specifically, these fitness claims by Nesse and Williams must be qualified with respect to the environment, because adaptation means adaptation to local environments. So, the fitness advantage that heterozygotes have over both sets of homozygotes is relative to the low-altitude environment in which malaria is present. If, however, people with each of these conditions were placed in an environment where no malaria existed and the altitude was very high, then the fitness advantages would change. Although the heterozygotes and the homozygote dominant people would still have a fitness advantage over the homozygote recessive people, the heterozygote people would no longer have a fitness advantage over the homozygote dominant people. The reason is that the heterozygote condition in high altitudes does not confer its propensity advantage in such places. That is, the high altitude causes the red blood cells to be modified. The result of this modification is hypoxia, which can produce fainting spells and other physically harmful conditions. In such a scenario, 
the homozygous dominant people would have a fitness advantage over the heterozygote people.

In general, heterozygote women are more prone to urinary tract infections than homozygote dominant women. Note that the extent to which the heterozygote condition is physically harmful with respect to exercise and other scenarios is unclear or controversial from the data collected and studied. (For the experiments and other physical ailments associated with the heterozygote condition, see Kark 2000 url.) Such details may further support the view that, from an evolutionary perspective, being "healthy enough" tells against strict optimality views regarding fitness - being ecologically bound bundles of compromises results in unwanted harmful side effects because selection can only work with variation that is available. Of course, if dual homeostasis is disrupted enough, then ascriptions of poor health are warranted in terms of PHJs.

Restated, in the low-altitude/malaria environment, the heterozygote people are healthier than both sets of homozygote people, because the evolved survival/reproduction propensity is present. Moreover, if it is true that there are side effects from the heterozygote condition even in this environment, then these people may still be somewhat unhealthy, but healthier than both homozygous people. The point is that adaptive traits need to be understood in relation to local environments - organisms, including humans, are ecologically bound entities - in making PHJs. The EM camp is sensitive to such nuances created by natural selection in ways that may not be emphasized or incorporated into the thinking of healthcare practitioners.

\section{Allergic Immunity}

Allergies are another set of conditions to consider with respect to EM (Ananth 2008). There are many types of allergies. They can be partially categorized as follows: (1) injected allergies (drugs, venom), (2) ingested allergies (foods), (3) inhaled allergies (pollen and animal dander), and (4) skin allergies (plants) (Profet 1991 and Barnes et. al. 1999). Allergies, which occur in varying degrees, are responses by the immune systems. In some cases, a minor allergic reaction can result in itchy eyes, mild sneezing, or slight inflammation of the tongue, having little or no serious effect on the dispositional properties of physiological homeostasis. The result is that an organism with a mild allergy is a relatively healthy organism. For example, some people are mildly or severely allergic to cat hair. In an environment where cats are present, people will be considered unhealthy or healthy to some degree, depending upon how their systems react to cats. Indeed, some people have no allergic reaction to cats. Of course, severe allergic reactions (e.g., bee stings in some people) can result in acute respiratory and pulmonary distress. In these sorts of cases, the organism is extremely unhealthy, because the dispositional properties of physiological homeostasis have been greatly reduced in the particular environment. Different sorts of allergies reveal that health is a state that not only admits of degree but may also admit of duration and vary with local environmental conditions. 
A further qualification about allergies is needed. Many allergic reactions are immune system responses governed by the immunoglobulin-E (IgE) system. Some have argued that allergy is a vestigial system that is beneficial in other species, but simply damages tissue in humans and should be viewed as an immune-response error. Thus, much like the appendix, the IgE system can cause physiological problems, but has no present function. In response, Profet (1991, pp. 24-25) has argued that the IgE system is a specialized evolved backup system to remove toxins from the body. As she notes, "The evolutionary persistence of the allergic capability, despite its physiological costs, implies the existence of an adaptive benefit for this capability that outweighs the costs; this undermines the view that allergy is an immunological error." The idea is that the body does have various toxin-fixing antibodies and enzymes that can decompose various sorts of chemical toxins. Yet, there are some toxins (e.g., venom, industrial pollutants, phenolic acids, and alkaloids) that are able to bypass these defenses. Profet argues that the IgE system is a second round of defense designed to eliminate these sorts of toxins that have evaded initial detection. In her own words (Profet 1991, p. 27), "[A]llergy is designed to be a last line of defense against toxins; that is, the allergic response is triggered when individual's primary antitoxin defense mechanisms have proven on a previous occasion to be insufficient in preventing a specific toxin from persisting in the bloodstream and damaging cells." Randolph Nesse and George Williams (1994, p. 163; bracketed addition mine) offer the following summary of Profet's theory about certain allergic reactions:

\footnotetext{
[An allergy] gets toxins out of you in a hurry. Shedding tears gets them [i.e., toxins] out of the eyes. Mucous secretions and sneezing and coughing get them out of the respiratory tract. Vomiting gets them out of the stomach. Allergic reactions act quickly to expel offending materials. This fits the rapidity with which toxins can cause harm. A few mouthfuls of those beautiful foxgloves in your garden can kill you a lot faster than a phone call can summon aid. Appropriately for Profet's theory, the only part of our immunological system that seems to be in a great hurry is that which mediates allergy. Other aspects of allergy that she mentions in support of her theory include the propensity to be triggered by venoms and by toxins that bind permanently to body tissue, the release of anticoagulants during allergic inflammation to counteract coagulant venoms, and the apparently erratic distribution of allergies to specific substances.
}

With respect to EM, allergies may seem problematic. For the IgE system has an evolved propensity to fight off certain toxins, it does so by disrupting homeostasis. That is, the $\operatorname{IgE}$ system is a biological function that can render an organism unhealthy.

The reply to the above difficulty is a reminder that evolutionary systems are not perfect systems. In an attempt to resolve one problem, biological systems can have disrupting side effects. This is simply the result of the body as a bundle of evolutionary compromises. With respect to homeostasis, the IgE system is an effector that has the evolved propensity of maintaining intercellular homeostasis. In order to do this, it must (to some degree) disrupt organism homeostasis at times. (A fever is an effector in much the same way.) According to EM, allergic responses (and fevers) 
can render an organism mildly or severely unhealthy (depending upon their degree of disturbance to intercellular and organism homeostasis) in the short run, so that both intercellular and organism homeostasis can be secured in the long run. This example stands as reminder that biological features that appear to reduce the health status of people may have evolutionary functions that are not obvious. These sorts of examples require that the PHJs that are made be qualified for short-term and longterm benefits. The EM perspective is respectful and alert to such scenarios. So, rather than telling against EM, allergies (i.e., biological functions that can cause harm) validate it.

\section{Concluding Remarks}

As The Origin of Species comes to its finish, Darwin (1859/1958, p. 458) renders the following energetic proclamation:

In the distant future I see open fields for far more important researches. Psychology will be based on a new foundation. . Much light will be thrown on the origin of man and his history.

It is interesting that Darwin chose to emphasize psychology as the "new" field of choice in anticipation of the fecundity he saw in his nascent ideas. For better or worse, evolutionary psychology has exploded, as Darwin envisioned, despite its arguably more delicate foundation (Buller 1999). Yet, despite its rather solid scientific underpinnings, EM is still awaiting for its Darwinian detonation to occur much to the chagrin of its proponents. On the modest appeal that EM can augment the reflection, practice, and training of medical practitioners and their cohorts, EM champions would, in the spirit of Darwin's affirmations, enthusiastically bellow that embracing the body as a bundle of evolutionary compromises could very well complement and point toward promising areas of research within medicine.

\section{Definition of Key Terms}

Evolutionary medicine

Evolutionary body

Physical health judgments discipline concerned with both biological and philosophical issues pertaining to the field of medicine and public health.

a bundle of adaptive compromises best understood as an ecologically oriented and evolutionary dynamic dual homeostatic properly functioning holistic system.

health and disease judgments pertaining to the physical human body made by 
Systemic Functionalism

Etiological Evolutionary Functionalism

Propensity Evolutionary Functionalism

Mixed Evolutionary Functionalism

Intercellular homeostasis

Organism homeostasis medical practitioners and public health officials.

a feature $\mathbf{X}$ has a function in system $\mathbf{S}$ if and only if activity $\mathbf{Y}$ of $\mathbf{X}$ causally contributes to S's overall capacity/performance of $\mathbf{Z}$.

a feature $\mathbf{X}$ currently has a function in an organism $\mathbf{O}$ if and only if activity $\mathbf{Y}$ of $\mathbf{X}$ produces effect $\mathbf{E}$ because $\mathbf{Y}$ and $\mathbf{E}$ were naturally selected (over some other causes and effects) to bring about the goals $\mathbf{G}$ of survival and reproductive success of $\mathbf{O}$.

a feature $\mathbf{X}$ has a function in an organism $\mathbf{O}$ by performing activity $\mathbf{Y}$ if and only if $\mathbf{Y}$ produces effect $\mathbf{E}$ because $\mathbf{Y}$ and $\mathbf{E}$ confer and will continue to confer a propensity $\mathbf{P}$ (within a certain range of environmental pressures) to bring about the goals $\mathbf{G}$ of survival and reproductive success of $\mathbf{O}$.

a feature $\mathbf{X}$ has a function in an organism $\mathbf{O}$ by performing an activity $\mathbf{Y}$ if and only if $\mathbf{Y}$ produces effect $\mathbf{E}$, and both $\mathbf{Y}$ and $\mathbf{E}$ confer a survival-enhancing propensity $\mathbf{P}$ on $\mathbf{O}$ (within a certain range of environmental pressures) and will continue to confer $\mathbf{P}$ on $\mathbf{O}$ (so long as a certain range of environmental pressures is present). And, moreover, $\mathbf{P}$ is currently present, because, ancestrally, there was natural selection in favor of retaining $\mathbf{P}$ to bring about the goals $\mathbf{G}$ of survival and reproduction. the internal state of an organism in which the stability of chemical composition, osmotic pressure, carbon dioxide, temperature, and quantity of waste with respect to intercellular remains constant. the overall state of an organism with respect to its behavioral and ecologically oriented activities in conjunction with intercellular homeostasis. 


\section{Summary Points}

1. Intro summary point: As a supplementary perspective to medical judgments, evolutionary medicine $[\mathbf{E M}]$ claims that it can assist in the theoretical, educational, and everyday practices of medical practitioners and public health officials.

2. Section II summary point: EM explores the philosophical, theoretical, and practical implications of incorporating an evolutionary perspective to the existing field of medicine.

3. Section III summary point: EM explores the importance of viewing the body as a "bundle of evolutionary compromises." From this perspective, medical practitioners are urged to embrace an evolutionary understanding of biological function and homeostasis as crucial to the understanding of the body as an ecologically oriented entity.

4. Section IV summary point: Some of the implications and overall efficacy of the EM conception of the human body are shown by an examination of some of the theoretical and practical approaches to (1) defense mechanism of fever, (2) genetic disease of sickle-cell anemia, and (3) the immunological response of allergies.

5. Section V summary: Charles Darwin was confident that an evolutionary perspective would open new areas of study with respect to the human animal. Although he specifies psychology as the prominent discipline that would blossom, contemporary advocates of EM would be delighted if he had included the field of medicine as well.

\section{References}

Alexander HK, Martin G, Martin OY, Bonhoeffer S (2014) Evolutionary rescue: linking theory for conservation and medicine. Evol Appl 7:1161-1179

Ananth M (2008) In defense of an evolutionary concept of health: nature, norms, and the human biology. Ashgate Press, Aldershot

Ananth M (2017) Bringing biology to life: an introduction to the philosophy of biology. Broadview Press, Calgary

APHA (American Public Health Association) 2016. https://www.apha.org/what-is-public-health

Ariew A, Cummins R, Perlman M (eds) (2002) Functions: new essays in the philosophy of psychology and biology. Oxford University Press, Oxford

Barnes KC, Armelagos GJ, Morreale SC (1999) Darwinian medicine and the emergence of allergy. In: Trevathan WR, Smith EO, McKenna JJ (eds) Evolutionary medicine. Oxford University Press, New York, pp 209-243

Basanta D, Miodownik M, Baum B (2008) The evolution of robust development and homeostasis in artificial organisms. Comput Biol 4:1-10. doi:10.1371/journal.pcbi.1000030

Bechtel W (1985) In defense of a naturalistic concept of health. In: Humber JM, Almeder RF (eds) Biomedical ethics review 1985. Humana Press, Clifton, pp 131-170

Bergman EM, Verheijen IWH, Van der Vlueten CPM, de Bruin ABH et al (2014) Influences on anatomical knowledge: the complete arguments. Clin Anat 3:296-303

Bernard C (1957) An introduction to the study of experimental medicine. Dover Publication, New York

Bigelow J, Pargetter R (1987) Functions. J Philos 84:181-196 
Bishai WR, Graham NMH et al (1996) Rifampin-resistant tuberculosis in a patient receiving rifabutin prophylaxis. N Engl J of Med 334:1573-1576

Boorse C (1987) Concepts of health. In: Van DeVeer D, Regan T (eds) Health care ethics: an introduction. Temple University Press, Philadelphia, pp 359-393

Boorse C (1997) A rebuttal on health. In: Humber JM, Almeder RF (eds) What is disease? Humana Press, Totowa, pp 1-134

Boorse C (2002) A rebuttal on functions. In: Ariew A, Cummins R, Perlman M (eds) Functions: new essays in the philosophy of psychology and biology. Oxford University Press, Oxford, pp $63-112$

Buller DJ (1999) Adapting minds: evolutionary psychology and the persistent quest for human nature. MIT Press, Cambridge

Cannon WB (1963) The wisdom of the body, revised and enlarged edn. W. W. Norton \& Company, New York

Cooper SJ (2008) From claude bernard to walter cannon. emergence of the concept of homeostasis. Appetite 51:419-427

Cummins R, Roth M (2010) Traits have not evolved to function the way they do because of a past advantage. In: Ayala FJ, Arp R (eds) Contemporary debates in the philosophy of biology. WileyBlackwell, West Sussex, pp 72-85

Darwin C (1859/1958) The origin of species by means of natural selection or the preservation of the favoured in the struggle for life, mentor edn. Mentor/Penguin Books, New York

Depew D (1998) Darwinism and developmentalism: prospects for convergence. In: van de Dijver G, Salthe SN, Delpos M (eds) Evolutionary systems: biological and epistemological perspectives on selection and self-organization. Kluwer, Dordrecht, pp 21-32

Dobzhansky T (1973) Nothing in biology makes sense except in the light of evolution. Am Biol Teach 35:125-129

Ewald PW (1994) Evolution of infectious disease. Oxford University Press, Oxford

Freeman S, Herron JC (2004) Evolutionary analysis, 3rd edn. Pearson/Prentice Hall, Upper Saddle River

Gilchrist GW, Joel G, Kingsolver JG (2001) Is optimality over the hill? In: Orzack SH, Sober E (eds) Adaptationism and optimality. Cambridge University Press, Cambridge, pp 219-241

Goldstein K (1963) The organism, a holistic approach to biology derived from pathological data in man. Beacon, Boston

Hardcastle V (1999) Understanding functions: a pragmatic approach. In: Hardcastle V (ed) Where biology meets psychology. The MIT Press, Cambridge, pp 27-43

Hartig T, Mitchell R, de Vries S, Frumkin H (2014) Nature and health. Annu Rev Public Health $35: 207-228$

Kark J (2000) Sickle cell trait. http://sickle.bwh.harvard.edu/sickle_trait.html

Keeney RE, Powley TL (2008) Body energy homeostasis. Appetite 53:442-445

McLaughlin P (2001) What functions explain. Cambridge University Press, Cambridge

Mayr E (1974) Teleological and teleonomic, a new analysis. Boston Stud Philos Sci 14:91-117

Mayr E (2000) Darwin's influence on modern thought. Sci Am 238:78-83

Millikan R (1993) White queen psychology and other essays for alice. MIT Press, Cambridge

Nesse R, Williamd GC (1994) Why we get sick: the new science of darwinian medicine. Times Book/Random House, New York

Nesse RM, Williams GC (1997) Evolutionary biology in the medical curriculum: what every physician should know. Bioscience 47:664-666

Nissen L (1997) Teleological language in the life sciences. Rowman \& Littlefield Publishers, Lanham

Odling-Smee JF, Laland KN, Feldman NW (2003) Niche construction: the neglected process in evolution. Princeton University Press, New Jersey

Older J (2004) Anatomy: a must for teaching the next generation. Surgeon 2:79-90

Papa V, Vaccarezza M (2013) Teaching anatomy in the XXI century: new aspects and pitfalls. Scientific World Journal 7281:1-5 
Perlman M (2010) Traits have evolved because of a past advantage. In: Ayala FJ, Arp R (eds) Contemporary debates in the philosophy of biology. Wiley-Blackwell, West Sussex, pp 53-71

Profet M (1991) The function of allergy: immunological defense against toxins. Quart Rev Biol 66:23-54

Roberts MBV (1986) Biology: a functional approach, 4th edn. ELBS with Nelson, UK

Salthe SN (1998) The role of natural selection theory in understanding evolutionary systems. In: van de Dijver G, Stanley N, Salthe SN, Delpos M (eds) Evolutionary systems: biological and epistemological perspectives on selection and self-organization. Kluwer, Dordrecht, pp 13-20

Seidel C (2002) Basic concepts in physiology. McGraw Hill, New York

Shanahan T (1999) Darwinian medicine. Lecture given to the biology department at loyola marymount university. http://philpapers.org/rec/SHADM

Sprouffke K, Merlo LMF, Garrish PJ, Maley CC, Sniegowski PD (2012) Cancer in light of experimental evolution. Curr Biol 22:762-771

Turney B (2007) Anatomy in a modern medical curriculum. Ann R Coll Surg Engl 89:104-107

Tyreman S J (2001) The concept of function in osteopathy and conventional medicine: a comparative study. Doctoral Dissertation, British School of Osteopath

World Health Organization (2007) Darwinian medicine: basic theory with practical uses for public health. http://www.who.int/global_health_histories/seminars/2005-2007/en/

Wouters A (2003a) Philosophers on function. Acta Biotheor 51:223-235

Wouters A (2003b) Four notions of biological function. Stud Hist Philos Biol Biomed Sci 34:633-668

Wright L (1976) Teleological explanations: an etiological analysis of goals and functions. University of California Press, Berkeley 